\title{
The Spineless-Aristapedia and Tango bHLH-PAS proteins interact to control antennal and tarsal development in Drosophila
}

\author{
Richard B. Emmons ${ }^{1}$, Dianne Duncan1, Patricia A. Estes ${ }^{2}$, Paula Kiefel' ${ }^{1}$, Jack T. Mosher², \\ Margaret Sonnenfeld ${ }^{2}$, Mary P. Ward ${ }^{2}$, lan Duncan ${ }^{1}$ and Stephen T. Crews ${ }^{2, *}$ \\ ${ }^{1}$ Department of Biology, Washington University, St. Louis, MO, USA \\ 2Department of Biochemistry and Biophysics, the University of North Carolina at Chapel Hill, Chapel Hill, NC 27599-7260, USA \\ *Author for correspondence (e-mail: steve_crews@unc.edu) \\ Accepted 11 June; published on WWW 5 August 1999
}

\section{SUMMARY}

The Drosophila spineless (ss) gene encodes a basic-helixloop-helix-PAS transcription factor that is required for proper specification of distal antennal identity, establishment of the tarsal regions of the legs, and normal bristle growth. $s s$ is the closest known homolog of the mammalian aryl hydrocarbon receptor (Ahr), also known as the dioxin receptor. Dioxin and other aryl hydrocarbons bind to the PAS domain of Ahr, causing Ahr to translocate to the nucleus, where it dimerizes with another bHLHPAS protein, the aryl hydrocarbon receptor nuclear translocator (Arnt). Ahr:Arnt heterodimers then activate transcription of target genes that encode enzymes involved in metabolizing aryl hydrocarbons. In this report, we present evidence that Ss functions as a heterodimer with the Drosophila ortholog of Arnt, Tango (Tgo). We show that the $s s$ and tgo genes have a close functional relationship: loss-of-function alleles of tgo were recovered as dominant enhancers of a ss mutation, and tgo-mutant somatic clones show antennal, leg, and bristle defects almost identical to those caused by $s s^{-}$mutations. The results of yeast twohybrid assays indicate that the $\mathrm{Ss}$ and Tgo proteins interact directly, presumably by forming heterodimers.
Coexpression of Ss and Tgo in Drosophila SL2 cells causes transcriptional activation of reporters containing mammalian Ahr:Arnt response elements, indicating that Ss:Tgo heterodimers are very similar to Ahr:Arnt heterodimers in DNA-binding specificity and transcriptional activation ability. During embryogenesis, Tgo is localized to the nucleus at sites of ss expression. This localization is lost in a ss null mutant, suggesting that Tgo requires heterodimerization for translocation to the nucleus. Ectopic expression of ss causes coincident ectopic nuclear localization of Tgo, independent of cell type or developmental stage. This suggests that the interaction of Ss and Tgo does not require additional signals, unlike the ligand-dependent interaction of $\mathrm{Ahr}$ and Arnt. Despite the very different biological roles of Ahr and Arnt in insects and mammals, the molecular mechanisms by which these proteins function appear to be largely conserved.

Key words: Arnt, Aryl hydrocarbon receptor, Dioxin receptor, Drosophila, homeotic gene, PAS domain, Antennal specification, Limb development, spineless-aristapedia, tango

\section{INTRODUCTION}

Loss-of-function mutations in the Drosophila spineless (ss) gene cause three distinct phenotypes: transformation of the distal antenna to distal second leg, deletion of most of the tarsal region in each leg, and reduction in the size of almost all bristles (Struhl, 1982; Lindsley and Zimm, 1992; Duncan et al., 1998). Consistent with these phenotypes, ss is expressed in the distal portion of the antennal imaginal disc, in the tarsal regions of the leg discs, and in bristle precursor cells (Duncan et al., 1998). ss is also expressed in the antennal segment, gnathal segments, limb primordia, and peripheral nervous system of the embryo. ss appears to be a primary determinant of distal antennal identity, as ectopic expression causes transformation of the distal leg, maxillary palp, and rostral membrane to antenna. In the legs, ss appears to function in the establishment of the tarsal primordia, as it is expressed only early in the tarsal region, and is required for later expression of the tarsal gene bric à brac (Duncan et al., 1998).

The protein encoded by $s s$ is a member of the basic-helixloop-helix-PAS domain (bHLH-PAS) family of transcription factors. PAS is named for the founding members of the family, Period, Aryl hydrocarbon receptor (Ahr) and Single-minded (Sim) (for review see Crews, 1998). PAS domains can function in dimerization (Huang et al., 1993), dimerization specificity (Pongratz et al., 1998), binding of aryl hydrocarbons (Dolwick et al., 1993) and interaction with non-PAS proteins (Coumailleau et al., 1995; Gekakis et al., 1995). Recently, it has become clear that several bHLH-PAS proteins share a common bHLH-PAS dimerization partner. In mammals, this is the so-called Aryl hydrocarbon receptor nuclear translocator, or Arnt. Arnt serves as the dimerization partner for Ahr and probably at least five other mammalian bHLH-PAS proteins (Crews 1998). These include the small family of proteins 
related to Hypoxia-inducible factor- $1 \alpha(\mathrm{HIF}-1 \alpha)$ that function in the physiological and developmental aspects of oxygen homeostasis (Semenza, 1998), and Sim1 and Sim2, the mammalian orthologs of Drosophila Sim. The Sim1 gene controls the formation of several hypothalamic nuclei (Michaud et al., 1998) and Sim2 has been implicated in Down Syndrome (Michaud and Fan, 1997). In Drosophila, a homolog of Arnt, called Tango (Tgo), has been shown to dimerize in vivo with the bHLH-PAS proteins Sim and Trachealess (Trh) (Sonnenfeld et al., 1997; Ohshiro and Saigo, 1997), and is probably also the dimerization partner for Similar, a protein highly related to HIF-1 $\alpha$ (Nambu et al., 1996).

Like Ss, Sim and Trh function to specify cell fate. Sim specifies midline cell identity in the CNS; in $\mathrm{sim}^{-}$mutants, midline cells are absent, whereas ectopic expression of sim can cause transformation of the entire CNS into midline cells (Thomas et al., 1988; Nambu et al., 1990, 1991). Trh controls development of the tracheae and salivary ducts (Isaac and Andrew, 1996; Wilk et al., 1996). These structures are absent in trh mutants, whereas ectopic tracheae can be induced by ectopic trh expression. Surprisingly, Sim:Tgo and Trh:Tgo heterodimers interact with the same target sequence, the CNS midline element (CME) (Wharton et al., 1994; Ohshiro and Saigo 1997; Sonnenfeld et al., 1997; Zelzer et al., 1997), which contains an ACGTG core. The effector specificities of Sim and Trh are determined by their PAS domains (Zelzer et al., 1997), which presumably interact differentially with other tissuespecific transcription factors.

The closest relative of Ss is the mammalian Ahr, also known as the dioxin receptor (reviewed in Hankinson, 1995; Schmidt and Bradfield, 1996; Rowlands and Gustafsson, 1997). Ss and Ahr have the same overall organization and are $71 \%$ identical in the bHLH region, $45 \%$ identical in the PAS domain, and $41 \%$ identical overall. Ahr binds toxic and carcinogenic compounds, and controls the transcription of genes that encode enzymes that metabolize these compounds. In the absence of ligand, Ahr exists in the cytoplasm complexed with Hsp90 (Denis et al., 1988) and Aryl hydrocarbon-interacting protein (Carver and Bradfield, 1997; Ma and Whitlock, 1997). Upon binding a membrane diffusible ligand such as dioxin, Ahr translocates to the nucleus where it dimerizes with Arnt (Pollenz et al., 1994). Ahr:Arnt heterodimers then activate transcription by binding to xenobiotic response elements (XREs), which contain a GCGTG core (Denison et al., 1988). Ahr:Arnt heterodimers do not interact with the CME element (for review of the DNA-binding specificities of bHLH-PAS heterodimers, see Schmidt and Bradfield, 1996).

In this paper, we employ genetic, molecular and cellular methods to assess the functional relationship between Ss and the Drosophila homolog of Arnt (Tgo). The first indication of a close relationship was the recovery of loss-of-function alleles of tgo in a screen for dominant enhancers of a ss mutation. Somatic clones homozygous for such tgo alleles show phenotypes that are almost identical to those shown by $s s^{-}$ clones, indicating that $\operatorname{tgo}$ is required for all of the major functions of ss. This observation suggested that Tgo might serve as the dimerization partner for Ss. Consistent with this, we show that Ss and Tgo interact directly in yeast two-hybrid assays. Moreover, coexpression of ss and tgo in Drosophila SL2 cells results in transcriptional activation of reporters carrying the XRE sequence. This suggests that Ss:Tgo heterodimers form in Drosophila, and recognize the same DNA sequence as Ahr:Arnt heterodimers. In the embryo, Tgo protein is localized to the nucleus in cells that express ss. This localization is lost in the $s s^{-}$mutant, whereas ectopic expression of ss causes coincident ectopic nuclear localization of Tgo. These observations support the conclusion (Ward et al., 1998) that nuclear localization of Tgo depends upon dimerization with a bHLH-PAS partner. This is in contrast to mammalian Arnt, which is nuclear in the absence of known partners (Pollenz et al., 1994). Our observations also indicate that dimerization of Ss and Tgo does not require any other spatially restricted factor, unlike the ligand-dependent interaction of $\mathrm{Ahr}$ and Arnt. Our results show that the Ahr:Arnt:XRE regulatory cassette is largely conserved in structure and mechanism between insects and vertebrates, despite its very different functions in these phylogenetic lineages.

\section{MATERIALS AND METHODS}

\section{Drosophila strains and transgenes}

The wild-type strain used was $w^{1118} . D f(3 R) s s^{D 114.4}$ and the ss hypomorphic $\left(s s^{D 114.7}\right)$ and null $\left(s s^{D 115.7}\right.$ and $\left.s s^{D 114.9}\right)$ alleles used are described by Duncan et al. (1998). The engrailed (en)-Gal4 and twist (twi)-Gal4 drivers were used as described previously by Ward et al. (1998). The UAS-ss transgene used contains a copy of the complete sscA5 cDNA clone coding sequence cloned into pUAST (Duncan et al., 1998).

\section{Generation of $\operatorname{tgo}$ somatic clones}

tgo alleles were placed in cis to $\mathrm{P}\left[r y^{+} ; h s-n e o\right.$; FRT $] 82 \mathrm{~B}(\mathrm{Xu}$ and Rubin, 1993). To generate the $M^{+}$clones shown in Fig. 1, hsFLP122 $f^{36 a}$; $\mathrm{P}\left[r^{+} ;\right.$hs-neo; FRT $] 82 \mathrm{~B}$ tgo/TM6B females were crossed to $\mathrm{P}\left[\mathrm{ry}^{+}\right.$; hs-neo; FRT $] 82 \mathrm{~B}$ M(3)95A Bsb/TM6, Ubx males. 48-hour egg lays were collected in glass vials, aged for 48 hours, and heat shocked at $37.5^{\circ} \mathrm{C}$ for 2 hours in a circulating water bath. For the clones described by Table 1 , hsFLP122 $f^{36 a}$; P $\left[r y^{+}\right.$; hs-neo; FRT $] 82 \mathrm{~B} P r$ $B s b / T M 6 B$ females were crossed to $\mathrm{P}\left[r y^{+} ; h s-n e o\right.$; FRT $] 82 \mathrm{~B}$ males, $\mathrm{P}\left[\mathrm{ry}^{+} ;\right.$hs-neo; FRT $] 82 \mathrm{~B}$ tgo $/ T M 6 B$ males, or $\mathrm{P}\left[r y^{+} ; h s-n e o\right.$; FRT]82B cu ss ${ }^{D 115.7 / T M 1}$ males. 24-hour egg lays were collected from each cross, aged 48 hours, and heat shocked as above. Adult progeny were pickled and their cuticles mounted as described by Duncan (1982).

\section{Yeast two-hybrid protein interaction analysis}

The yeast two-hybrid system of Brent and coworkers (Brent, 1994) was utilized. The Ss bait used was a LexA-Ss fusion protein containing the Ss bHLH and PAS domains, but not the C-terminal polyglutamine sequences. The plasmid encoding this bait was constructed by cloning a 377-codon fragment of the sscA6 cDNA (Duncan et al., 1998) extending from the initiating ATG to an internal NcoI site into pEG202 (Brent, 1994). The Ss prey-encoding plasmid was constructed by cloning a 670 codon fragment of sscA6 extending from the initiator ATG to an internal XhoI site into pJG4-5 (Brent, 1994). The Sim, Trh, and Tgo preys have been described previously (Sonnenfeld et al., 1997). Sim and Trh preys include bHLH and PAS domains and lack C-terminal regions, whereas the Tgo prey includes the full length Tgo sequence. The Drosophila Septin1 protein (Fares et al., 1995) was used as a negative control, since it is not anticipated to dimerize with Ss. The experiments were not repeated switching bait and preys, since all Tgo baits tested self-activate.

Bait and prey plasmid combinations were co-transformed into EGY48, which contains the pSH18-34 lacZ reporter gene preceded by eight LexA binding sites. Three independent colonies were picked 
from each transformation, and assayed individually for $\beta$ galactosidase activity in a standard enzyme assay using the chromogenic substrate o-nitrophenyl- $\beta$-D-galactoside (ONPG).

\section{SL2 cell culture transient expression assays}

The ss expression plasmid, pAct-ss, was generated by cloning an $E c o$ RI fragment of the sscA5 cDNA clone, which contains the entire ss coding sequence, into the EcoRI site of pAct5CSRS (Burtis and Baker, 1989; Han et al., 1989). The construction of pAct-sim and pAct-tgo are described by Sonnenfeld et al. (1997). The reporter genes contained multimerized XREs and CMEs cloned into the pGL3 enhancer tester vector (Promega) that utilizes a firefly luciferase (luc) reporter gene. XRE-luc contains five copies in the same orientation of the oligonucleotide AATTCTCTTCTCACGCAACTCCGGGGC, which corresponds to an Ahr:Arnt XRE binding site in the human CYP1A1 cytochrome P450IA1 gene (Pongratz et al., 1991). CME-luc has six copies of the Toll ( $T l$ ) site 4 CME (Wharton et al., 1994; Sonnenfeld et al., 1997) cloned into pGL3. The copia-LTR-luc transfection control plasmid consists of the copia LTR promoter driving the renilla luc gene in the pGL3-Basic vector (Promega). Transient transfections in Drosophila SL2 cells were carried out using the calcium phosphate method (Fehon et al., 1990). Each transfection was done at least three times using $5 \mu \mathrm{g}$ DNA for each reporter plasmid and expression plasmid. The copia-LTR-luc control plasmid was present in $2.5 \mu \mathrm{g}$, and pAct5CSRS was added, when needed, to achieve a final DNA amount of $17.5 \mu \mathrm{g}$. After 48 hours of growth, firefly and renilla luc expression was assayed using the Dual Luciferase Assay (DLA; Promega).

\section{Immunostaining and in situ hybridization of embryos}

Antibody staining of embryos was carried out according to standard protocols (Patel et al., 1987). Staining with murine monoclonal antiTgo and rat polyclonal anti-Trh, and visualization by confocal microscopy was as described by Ward et al. (1998). Biotinylated antimouse secondary antibody was followed by HRP-avidin (Vector Labs) and FITC-conjugated tyramide (TSA Direct, NEN). The anti-rat secondary antibody was conjugated with Texas Red (Molecular Probes). In situ hybridization of whole-mount embryos was carried out using a full length ss digoxigenin-labeled antisense RNA probe, followed by alkaline phosphatase/X-phosphate/NBT histochemistry (Tautz and Pfeiffle, 1989).

\section{RESULTS}

\section{Isolation of $\boldsymbol{t g o}$ alleles as enhancers of $\boldsymbol{s s}$}

The ss gene is dose-sensitive; animals heterozygous for ss deficiencies show swelling and development of ectopic bristles in the basal cylinder, located at the base of the arista (Fig. $1 \mathrm{~A}, \mathrm{~B})$. Also, one of the aristal branches is often transformed to a socketed bristle. These effects presumably reflect a weak transformation to tarsus. To identify genes that interact with $s s$, we screened for dominant enhancement of this transformation in heterozygotes for the $s s$ deficiency $D f(3 R) s s^{D 114.4}$. Enhancer mutations were recovered in several genes, including tgo. Three $\operatorname{tgo}$ alleles, designated $\operatorname{tgo}^{5}, \operatorname{tg}^{6}$ and $\operatorname{tgo}{ }^{7}$, were recovered. These fail to complement one another and the $\operatorname{tgo}{ }^{1}$, $\operatorname{tgo}^{2}$, and $\operatorname{tgo}^{3}$ alleles of Sonnenfeld et al., (1997). Subsequent tests of $\operatorname{tgo} o^{1}, \operatorname{tgo}^{2}$ and $\operatorname{tgo} o^{3}$ show that these also cause dominant enhancement of the antennal transformation in $D f(3 R) s s^{D 114.4}$ heterozygotes. $D f(3 R) s s^{D 114.4} /$ tgo heterozygotes show numerous bracted bristles (characteristic of leg identity) in distal AIII and the basal cylinder, and swelling of the base of the arista (Fig. 1C).

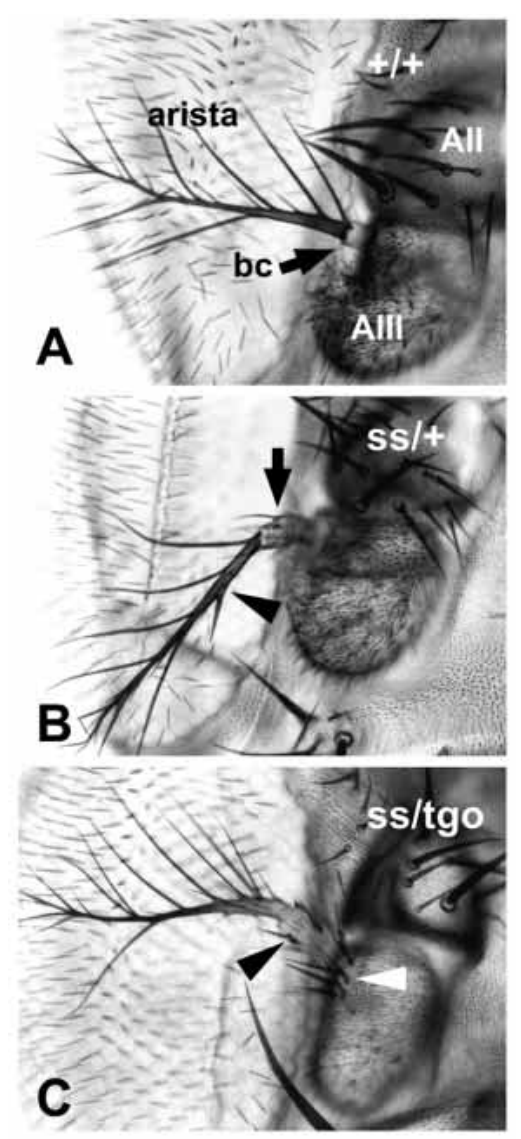

Fig. 1. tgo alleles enhance the dosage sensitivity of ss. (A) Wild-type (Oregon-R) antenna showing segments AII and AIII with arista extending from the basal cylinder (bc; arrow). (B) Antenna from a $D f(3 R) s s^{D 114.4} /+$ heterozygote. Note bristles on the basal cylinder (arrow), and socketed bristle on arista (arrowhead) indicating weak ss phenotype. (C) Antenna from a $D f(3 R) s s^{D 114.4} /$ tgo $^{5}$ heterozygote showing enhanced ss phenotype. Note swelling at base of arista, and the presence of bracted bristles on the basal cylinder (black arrowhead) and distal AIII (white arrowhead).

\section{Somatic clones of tgo mutants show ss phenotypes}

Since existing tgo alleles are lethal when homozygous, we have assessed the imaginal requirements for tgo by examining the adult cuticular phenotypes of somatic clones homozygous for $\operatorname{tgo}$ alleles. We find that such clones show defects almost identical to those seen in $s s^{-}$animals (Fig. 2; Table 1). These include transformation of distal antenna to distal leg, deletion of distal structures in the legs and maxillary palps, extension of the wing perpendicular to the body, and reduction in the size of almost all bristles. Somatic clones were generated for $\operatorname{tgo} o^{1}, \operatorname{tgo}^{5}$, and $\operatorname{tg} o^{6}$. The results suggest that $\operatorname{tgo}^{5}$ is close to a null allele, $\operatorname{tgo}^{l}$ is intermediate, and $\operatorname{tgo}^{6}$ is a weak allele.

\section{Antenna}

Like ss alleles, the tgo alleles tested have no effect in the proximal antennal segments (AI and AII). However, $\operatorname{tgo}^{1}$ clones transform the distal portion of AIII and the arista to a well-segmented tarsus that usually has a partially formed claw (Fig. 2A,B). The proximal portion of AIII is not affected. This phenotype is very similar to that caused by hypomorphic 
Fig. 2. Somatic clones of tgo show ss phenotypes. All clones were induced in a FRT82B M(3)95A Bsb background with the exception of the abdominal clones, which were induced in a FRT82B Pr Bsb background. (A-E) tgo and $s s$ phenotypes in the antenna.

(A) Control antenna from a FRT82B M(3)95A Bsb heterozygote. (B) tgo $^{l}$ clone that includes AI through the arista. Distal AIII, the basal cylinder, and the arista are transformed to a well segmented tarsus terminating in a claw. (C) Antenna from a $s s^{a}$ mutant genotype $\left(s^{s D 114.7} / s^{D 115.7}\right)$ showing an almost identical phenotype to that in B.

(D) $\operatorname{tg} o^{5}$ clone that includes AII through the arista. Distal AIII, the basal cylinder, and the arista are transformed to tarsus. However, unlike B and C, tarsal segmentation is not seen. (E) Antenna from a homozygote for the ss null allele $s s^{D 115.7}$. Note deletion of tarsal segments relative to $\mathrm{C}$, and loss of the identity of AIII (arrowhead). (F-I) tgo and ss phenotypes in the leg. All legs are oriented with proximal to the top and ventral to the right. (F) Control leg from a FRT82B M(3)95A Bsb heterozygote. Tarsal segments are labeled T1-T5. In G and $\mathrm{H}$, legs are shown in which tgo mutant clones fill both anterior and posterior compartments. (G) tgo $^{1}$ leg. Tarsal segments are shortened, and a partial fusion of T4 and T5 is present (arrowhead). (H) $\operatorname{tgo}^{5} \mathrm{leg}$. Arrowhead indicates fusion of distal T1 to proximal T5. (I) Leg from a ss null mutant ( $s s^{D 115.7}$ ) homozygote, showing a tarsal deletion similar to H. (J-M) The effects of tgo and ss mutants on abdominal macrochaetes. Each panel shows the posterior edge of the fourth tergite. (J) Wild-type clone induced in a FRT82B Pr Bsb background. The clone includes two macrochaetes (arrowheads), large bristles present at the posterior edge of the tergite pigment band. These bristles are normally completely absent in the $\operatorname{Pr} B s b$ background, although the socket remains intact (see white arrowhead in K). (K) $\operatorname{tgo}^{1}$ clone. Macrochaetes are smaller than in wild type (arrowheads). (L) $\operatorname{tgo}^{5}$ clone. Macrochaetes (arrowheads) are smaller than in $\operatorname{tgo}^{1}$ clones. (M) An $s s$ null mutant $\left(s s^{D 115.7}\right.$ ) clone showing very tiny $s s^{-}$ macrochaetes (arrowheads). (N-P) Effects of tgo and $s s$ in the maxillary palp. (N) Control palp from a FRT82B M(3)95A Bsb heterozygote. (O) $\operatorname{tgo}^{5}$ clone. Note truncation of palp. (P) Palp from a ss null mutant $\left(s s^{D 115.7}\right)$ homozygote.

alleles of $s s$ ( $s s^{a}$ alleles) (compare Fig. 2B,C). $\operatorname{tgo}^{6}$ clones show a similar, but much weaker, transformation (data not shown). $\operatorname{tgo}^{5}$ clones transform distal AIII and the arista to a tarsus that is deleted for most of its segments (Fig. 2D). This phenotype is similar to that of ss null mutants (Fig. 2E). However, unlike ss null mutants, arista-claw intermediates are often present, and the identity of proximal AIII is not affected.

\section{Leg and maxillary palp}

Distal leg clones homozygous for $\operatorname{tgo}^{5}$ show pattern deletions that often include the second through fourth tarsal segments (Fig. 2F,H). Such deletions are very similar to those caused by $s s^{-}$alleles (compare Fig. 2H,I). $\operatorname{tgo}^{1}$ clones show much weaker deletions (Fig. 2G), and $\operatorname{tg}^{6}$ clones do not visibly affect distal leg structures. None of the tgo alleles tested has any effect in proximal regions of the leg. Like $s s^{-}$alleles, $\operatorname{tgo}^{5}$ also causes distal deletions in the maxillary palps (Fig. 2N,O). $\operatorname{tgo}^{1}$ clones show weaker deletions in the palp, and $\operatorname{tgo}^{6}$ clones appear normal (data not shown).

\section{Wings}

For all three tgo alleles tested, homozygous clones in the winghinge region cause the wing to be held out away from the body (data not shown). Strong alleles of ss cause this same phenotype.

\section{Bristles}

$\operatorname{tgo}^{5}$ clones show a strong reduction in bristle size (Fig. 2J,L). However, this reduction is less severe than that caused by $s s$ null alleles (Fig. 2M). $\operatorname{tgo}^{1}$ has an intermediate effect on bristle size (Fig. 2K), and $\operatorname{tgo}^{6}$ has a very weak effect (data not shown).

\section{Other structures}

To determine whether tgo is required for patterning cuticular structures not affected by $s s$, we have examined $\operatorname{tgo} o^{5}$ clones in the proboscis, vibrissae, dorsal head, dorsal thorax, wings and abdomen. With the exception of a reduction in bristle size, $\operatorname{tgo}^{5}$ clones appear normal in all of these locations. Moreover, the 


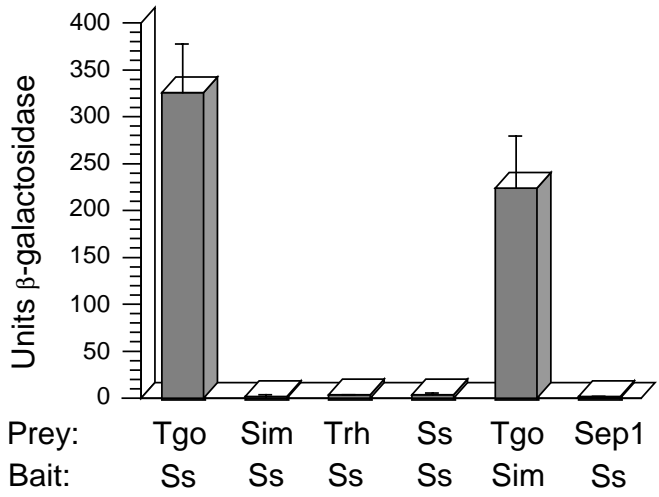

Fig. 3. Yeast two-hybrid interaction assays show that Ss dimerizes with Tgo. Liquid cultures of EGY48 co-transformed with the Prey and Bait combinations shown were assayed in the presence of galactose for $\beta$-galactosidase activity using the ONPG assay. The reporter is the pSH18-34 lacZ gene. Data is expressed in units $\beta$ galactosidase activity \pm s.e.m. All bHLH-PAS protein baits and preys contain intact bHLH and PAS domains, but all lack C-terminal activation domain sequences except Tgo prey, which is full length. Drosophila Septin 1 (Sep1) is a cytoplasmic protein involved in cytokinesis, and functions as a negative control.

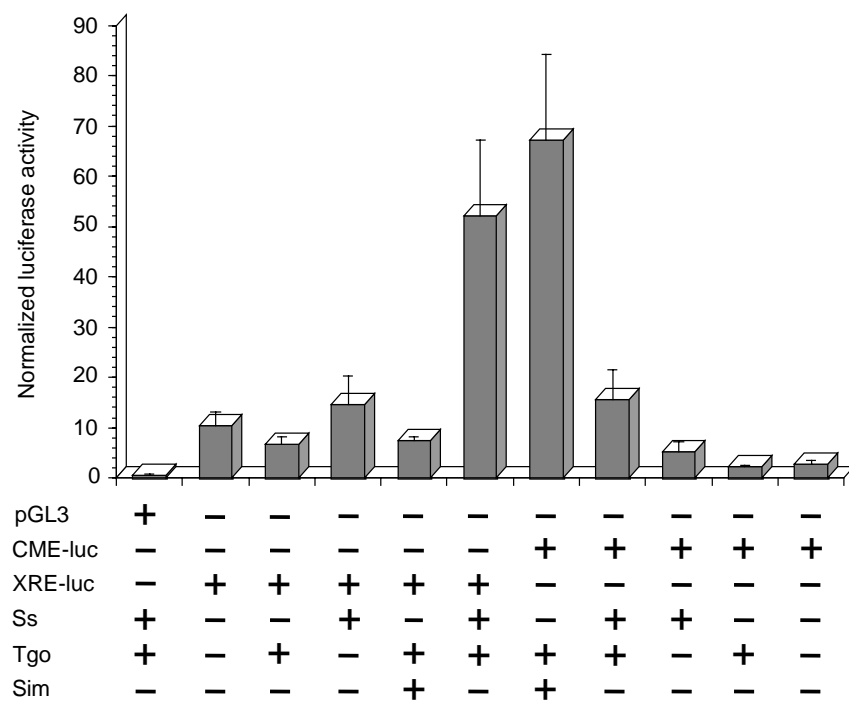

Fig. 4. Drosophila cell culture experiments show that Ss and Tgo interact, bind DNA, and activate transcription from XRE-bearing reporter genes. Drosophila SL2 cells were transiently transfected with: expression plasmids that act as sources of Ss and Tgo protein, reporter genes, and a transfection normalization reporter. The expression plasmids are pAct-ss, pAct-sim and pAct-tgo. The reporter plasmids, all of which have a multimerized DNA binding element that drives firefly luc from pGL3, are: XRE-luc, which contains 5 copies of the cytochrome P450IA1 XRE in the same orientation, and CME-luc that contains 6 copies of the $T l$ gene site 4 CME in the same orientation. The enhancerless control is pGL3, which contains the same promoter and $l u c$ reporter gene as XRE-luc and CME-luc, but lacks XRE or CME sequences. The copia-LTR-luc plasmid that has a renilla luciferase gene was used to normalize transfection efficiency between different transfections. Normalized luciferase units are expressed as arbitrary units as the mean \pm s.e.m. of 3 independent transfections.
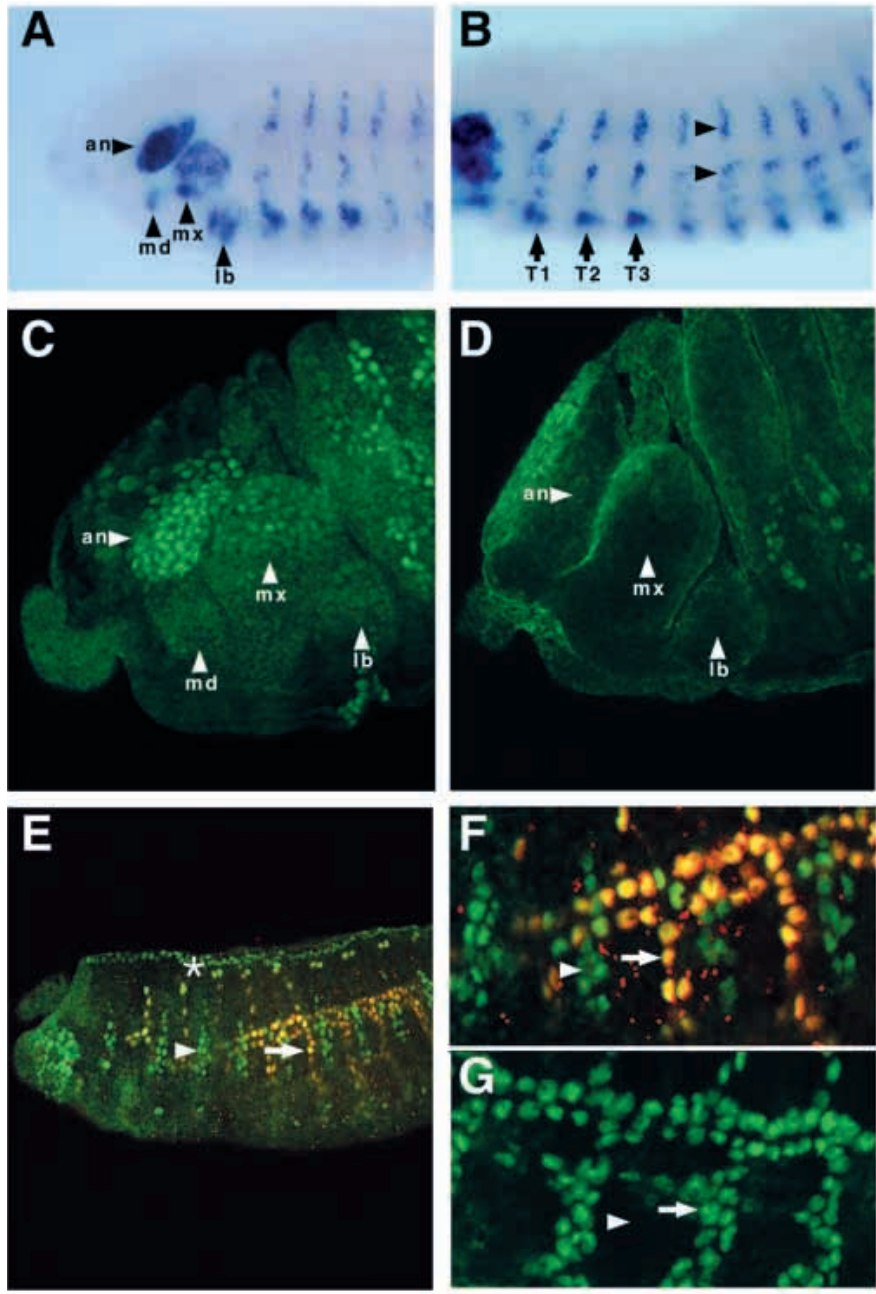

Fig. 5. Tgo nuclear accumulation correlates with ss expression. All embryos are wild-type except those shown in D and G; dorsal points to the top and anterior to the left. (A) In situ hybridization of an ss probe hybridized to a stage 14 embryo. Strong staining is observed in the larval antennal anlage (an; arrowhead). Weaker staining is seen in the mandibular (md), maxillary, (mx), and labial (lb) segments (arrowheads). (B) In situ hybridization of ss probe to a stage 15 embryo showing ss expression in the leg anlage (arrows) found in the ventral regions of the T1-T3 segments, and in body wall sensory cells (arrowheads). (C) Anti-Tgo staining of a stage 14 embryo showing strong nuclear accumulation in the antennal anlage (an), and weak nuclear staining in the mandibular ( $\mathrm{mn})$, maxillary $(\mathrm{mx})$, and labial (lb) segments (arrowheads). (D) Staining of a stage $14 s s^{D 114.9}$ mutant embryo with anti-Tgo showing an absence of Tgo nuclear accumulation in the antennal, mandibular and labial segments (arrowheads). (E) Double staining of a stage 15 embryo with anti-Tgo (FITC; green) and anti-Trh (Texas Red; red). The yellow cells contain both Tgo and Trh, and are tracheal cells (arrow). The green, segmentally repeated cells with strong nuclear Tgo accumulation are Ss-positive sensory cells (arrowhead). There is also strong nuclear Tgo accumulation in cells of the primordial dorsal vessel $(*)$.

(F) Higher magnification of a stage 15 embryo double stained for Tgo (green) and Trh (red) showing sensory cell Tgo nuclear accumulation (arrowhead) and double-stained (yellow) tracheal cells (arrow). (G) Staining of a stage $15 s s^{D 114.9}$ mutant embryo with anti-Tgo (green) shows tracheal cell nuclear accumulation, but sensory cell Tgo nuclear accumulation is absent. Arrowhead indicates region in a segment where sensory cell nuclear Tgo staining is found in wild-type embryos, and arrow indicates tracheal Tgo nuclear accumulation. 
Table 1. Quantitative analysis of $\operatorname{tgo}$ and $s s$ clones in the adult cuticle

\begin{tabular}{|c|c|c|c|c|c|c|c|c|c|}
\hline & \multicolumn{3}{|c|}{+} & \multicolumn{3}{|c|}{$\operatorname{tgo} 5$} & \multicolumn{3}{|c|}{$s s^{D 115.7}$} \\
\hline & no. clones & $n$ & $\%$ affected & no. clones & $n$ & $\%$ affected & no. clones & $n$ & $\%$ affected \\
\hline Distal antenna & $38^{\mathrm{a}}$ & 70 & 0 & 110 & 136 & $100^{c}$ & 61 & 94 & $100^{c}$ \\
\hline Distal leg & 146 & 175 & $5^{\mathrm{b}}$ & 111 & 178 & $100^{\mathrm{d}}$ & 86 & 197 & $100^{\mathrm{d}}$ \\
\hline Maxillary palp & 34 & 70 & 0 & 26 & 136 & $92^{\mathrm{e}}$ & 31 & 94 & $100^{\mathrm{e}}$ \\
\hline Wing margin & 130 & 68 & 0 & 81 & 78 & 0 & 117 & 76 & 0 \\
\hline Proximal antenna & 67 & 70 & 0 & 71 & 136 & 0 & 42 & 94 & 0 \\
\hline Proximal leg & 551 & 175 & 0 & 341 & 178 & 0 & 309 & 197 & 0 \\
\hline Proboscis & 40 & 35 & 0 & 42 & 68 & 0 & 30 & 47 & 0 \\
\hline Vibrissae & 51 & 35 & 0 & 79 & 68 & 0 & 55 & 47 & 0 \\
\hline Dorsal head & 78 & 35 & 0 & 102 & 68 & 0 & 51 & 47 & 0 \\
\hline Dorsal thorax & 127 & 34 & 0 & 134 & 39 & 0 & 156 & 38 & 0 \\
\hline Abdomen & 240 & 34 & 0 & 252 & 33 & 0 & 230 & 39 & 0 \\
\hline
\end{tabular}

frequencies of $\operatorname{tgo}^{5}$ and $s s^{-}$clones are comparable in these tissues, as well as in those affected by $s s$ (see Table 1). Taken together, our results show that the requirements for tgo and ss in the adult cuticle are almost identical.

\section{Interaction assays show that Ss dimerizes with Tgo}

The ability of Ss to form dimers with Tgo was tested directly using the yeast two-hybrid interaction assay (Fields and Song, 1989; Brent, 1994). The coding sequence of Ss containing the bHLH and PAS domains, but lacking the polyglutaminecontaining putative transcriptional activation sequences, was fused to LexA to create a bait protein. This bait was tested against Tgo, Sim, Trh and Ss bHLH-PAS protein preys, as well as Drosophila Septin1, which acts as a negative control. The results indicate that Ss interacts strongly with Tgo, but does not form dimers with Sim or Trh (Fig. 3). It is also unable to form homodimers. The strength of the Ss:Tgo interaction is comparable to that observed between Sim and Tgo (Sonnenfeld et al., 1997).

\section{Transient expression studies in Drosophila tissue culture indicate that Ss:Tgo heterodimers activate transcription from XRE binding sites}

To test whether Ss and Tgo can interact and activate transcription, we carried out transient transfection assays in Drosophila SL2 cells using XRE and CME-containing reporters. Neither Ss nor Tgo significantly activate the CMEluc or XRE-luc reporters when transfected alone (Fig. 4). However, when cotransfected, Ss and Tgo strongly activates the XRE-luc reporter. Cotransfection of ss and tgo only causes weak activation of the CME-luc reporter. In contrast, cotransfection of sim and tgo causes strong activation of the CME-luc reporter, but does not significantly activate the XRE$l u c$ reporter. When compared directly, Ss:Tgo heterodimers are over 3-fold more active with XRE-luc compared to CME-luc, whereas Sim:Tgo heterodimers are almost 10-fold more active with CME-luc compared to XRE-luc. Taken together, these assays indicate that Ss and Tgo can dimerize, enter nuclei, bind the XRE sequence, and activate transcription in Drosophila cells. The results also demonstrate that Ss:Tgo heterodimers, like Ahr:Arnt (Schmidt and Bradfield, 1996), prefer XRE sequences over CME sequences.

\section{Embryonic nuclear localization of Tango correlates with sites of ss expression}

In the embryo, Tgo is cytoplasmic in some cells and nuclear in others (Ward et al., 1998). In previous work, it was shown that Tgo is nuclear in the CNS midline and tracheal cells, which are the sites of sim and trh expression (Ward et al., 1998). Here, we show that Tgo is also nuclear in cells that express $s s$. In the embryo, ss is expressed in the antennal segment, the gnathal segments, the leg anlage, and the peripheral nervous system (Fig. 5A,B; Duncan et al., 1998). Strong nuclear accumulation of Tgo is seen in the antennal segment (Fig. 5C), which expresses the highest level of ss (Fig. $5 \mathrm{~A})$. Nuclear accumulation of Tgo is also observed in the gnathal segments (mandibular, maxillary, and labial), but the intensity of staining is relatively weak compared to the antennal segment (Fig. 5C). This correlates with the relatively weak expression of ss in the gnathal segments when compared to the antennal segment (Fig. 5A; Duncan et al., 1998). Nuclear localization of Tgo in the antennal and gnathal segments is dependent on ss, as it is not seen in a ss null mutant (Fig. 5D). The expression of $s s$ in the appendage primordia and the peripheral nervous system (Fig. 5B) also correlates with Tgo nuclear accumulation (Fig. 5E-G). Sensory cells that express $s s$ are in close proximity to the tracheal cells that express $t r h$. To distinguish these, embryos were labeled with anti-Trh and anti-Tgo (Fig. 5E,F). Non-tracheal cells that show nuclear Tgo are observed in the location of $s s$-expressing sensory cells. This non-tracheal Tgo nuclear accumulation is absent in ss mutant embryos (Fig. 5G). These results indicate that Tgo accumulates in the nuclei of ss-expressing antennal, gnathal and sensory cells, consistent with the formation and nuclear accumulation of Ss:Tgo heterodimers in vivo. Surprisingly, we do not observe significant Tgo nuclear accumulation in the limb primordia, even though ss is expressed in these cells. This may reflect regulatory events idiosyncratic to the limb primordia, or 

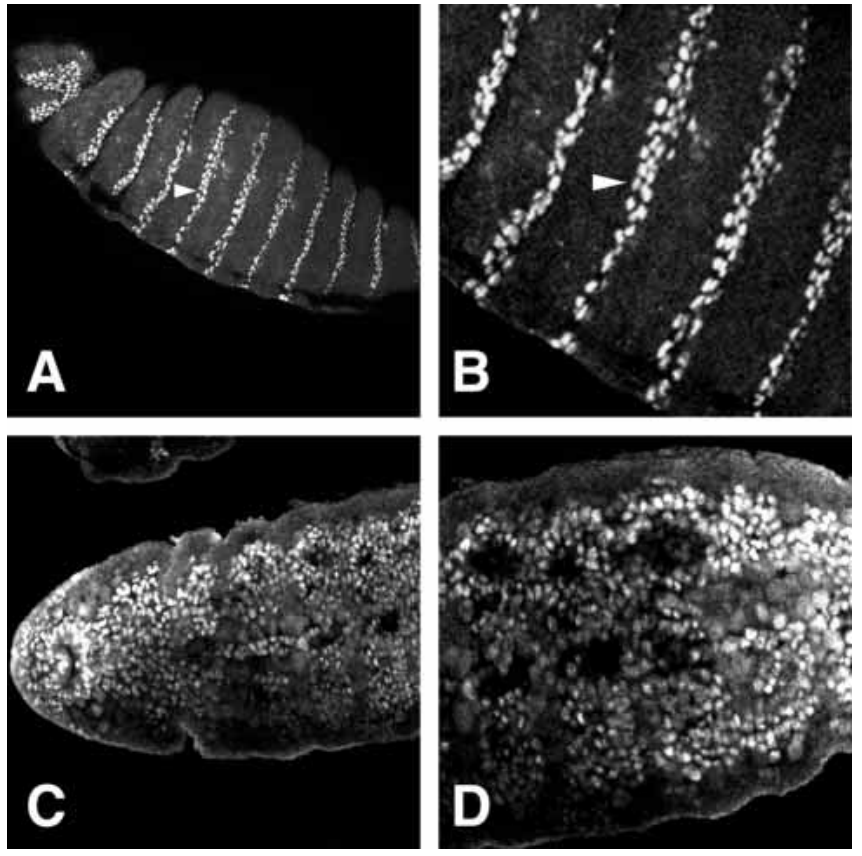

Fig. 6. Ectopic expression of ss results in ectopic nuclear Tgo accumulation. All embryos have anterior to the left. (A) Sagittal view of a stage 14 en-Gal4/UAS-ss embryo stained with anti-Tgo showing accumulation of Tgo in en stripes. (B) Higher magnification of the embryo shown in A revealing that Tgo is localized to nuclei in the en stripes. (C) Ventral view of a stage 11 twi-Gal4/UAS-ss embryo stained with anti-Tgo showing that Tgo accumulates in the mesoderm. (D) Higher magnification of embryo shown in $\mathrm{C}$ revealing the nuclear localization of mesodermal Tgo.

a lack of sensitivity of the immunostaining, since the limb primordia express $s s$ at considerably lower levels than the antennal segment. Tgo nuclear accumulation was also observed in the cells of the dorsal vessel (Fig. 5E). Since, sim, ss and trh are not expressed in the dorsal vessel, an additional bHLH-PAS protein may function in combination with Tgo in controlling the development or physiology of these cells, which comprise the Drosophila circulatory system.

When ectopic expression of a UAS-ss transgene was driven by en-Gal4, Tgo was found to accumulate in nuclei in circumferential ectodermal en stripes (Fig. 6A,B). Similarly, expression of $s s$ in mesodermal cells (driven by twi-GAL4) caused nuclear accumulation of Tgo in the mesoderm (Fig. 6C,D). These experiments support the conclusion that Ss and Tgo interact in vivo, and suggest that their interaction and nuclear accumulation does not depend on additional, spatiallyrestricted, factors.

\section{DISCUSSION}

\section{Ss functions as a heterodimer with Tgo}

In this report we present several lines of evidence that the Ss bHLH-PAS protein functions as a heterodimer with Tgo, the Drosophila homolog of mammalian Arnt. A close functional relationship between $s s$ and $\operatorname{tgo}$ was indicated by our recovery of loss-of-function alleles of tgo in a screen for dominant enhancers of ss. We show that three previously identified tgo alleles (Sonnenfeld et al., 1997) also enhance ss loss-offunction phenotypes. Three tgo alleles were examined to determine their phenotypes in homozygous clones in the adult cuticle. The strongest allele tested $\left(\operatorname{tg} O^{5}\right)$ caused phenotypes almost identical to those found in $s s^{-}$adults or in $s s^{-}$clones. These phenotypes include transformation of distal antenna to leg, deletion of most of the tarsal region, truncation of the maxillary palps, reduction in the size of almost all bristles, and extension of the wings away from the body. However, two differences were noted between the effects of $\operatorname{tgo}^{5}$ and $s s$ null alleles: $\operatorname{tgo}^{5}$ clones do not affect the proximal portion of AIII, whereas $\mathrm{ss}^{-}$animals show a complete loss of AIII identity; and $\operatorname{tgo}^{5}$ clones show a weaker reduction in bristle size than seen in $s s^{-}$animals. These differences could result from residual activity of the $\operatorname{tgo}^{5}$ allele, or might reflect weak $\operatorname{tgo}$ independent functions of ss.

Because Ahr (an ortholog of Ss) functions as a heterodimer with Arnt in mammalian cells, and because Tgo is known to be a dimerization partner for two other bHLH-PAS proteins (Sim and Trh), our working hypothesis at the outset was that Ss functions as a heterodimer with Tgo. The finding of almost identical requirements for tgo and $s s$ in the adult cuticle is consistent with this hypothesis. To test for direct interaction of Ss and Tgo, we carried out a series of yeast two-hybrid assays. These demonstrate that Ss and Tgo interact, and show that their association requires only the bHLH and PAS domains of Ss. This suggests that dimerization of Ss and Tgo is similar to that of Ahr and Arnt (Reisz-Porszasz et al., 1994; Lindebro et al., 1995). Ss only interacted with Tgo. Ss does not homodimerize nor does it interact with any other bHLH-PAS protein tested. Similar results were also observed previously for Sim, Trh and Ahr (Sonnenfeld et al., 1997); they only interacted with Tgo or Arnt. We also show that coexpression of Ss and Tgo in transient transfection assays in Drosophila SL2 cells results in transcriptional activation of reporters containing multiple XREs, the binding sites recognized by Ahr:Arnt heterodimers in mammalian cells. Cells singly transfected with ss or tgo do not show activation of these same reporters. Thus, it would appear that Ss and Tgo interact in SL2 cells, presumably by dimerization, localize to the nucleus, bind the XRE sequence, and activate transcription.

Additional evidence that Ss and Tgo interact is provided by our finding that Tgo is localized to cell nuclei at sites of ss expression. In embryos, $s s$ is expressed prominently in the antennal segment gnathal segments, and the peripheral nervous system. Nuclear accumulation of Tgo occurs in these locations in wild type, but not in $s s^{-}$embryos. Also, we find that ectopic expression of $s s$ driven by en-GAL4 or twi-GAL4 causes coincident ectopic nuclear localization of Tgo. These observations suggest that nuclear localization of $\mathrm{Tgo}$ is promoted by heterodimerization with Ss. Ward et al. (1998) have presented similar observations suggesting that Tgo nuclear localization is also promoted by dimerization with the Sim and Trh bHLH-PAS proteins.

To summarize, we present four main lines of evidence that Ss functions as a heterodimer with Tgo. First, we find almost identical requirements for tgo and $s s$ in the adult cuticle. Second, we show that Ss and Tgo interact directly in a yeast two-hybrid assay. Third, we show that ss and tgo activate transcription of an XRE reporter after transient cotransfection of Drosophila SL2 cells. Fourth, we find that Tgo becomes 
localized to the nucleus at sites of $s s$ expression in the embryo.

\section{Functional divergence of the Ahr:Arnt:XRE regulatory cassette}

The Drosophila Ss protein is the closest known relative of mammalian Ahr (Duncan et al. 1998). These proteins share extensive sequence identity, especially in their bHLH regions, and must share common ancestry, as several of the splice sites in the $s s$ and $A h r$ genes are precisely conserved. In this report, we show that Ss and Ahr also share two important functional properties; both function as heterodimers with an homologous partner (Arnt/Tgo), and both appear to recognize the same DNA sequence, the XRE. Although the target sequence recognized by Ss:Tgo heterodimers in vivo has not been defined, we show here that, like Ahr:Arnt heterodimers, these can activate transcription from XRE containing reporters in cell culture.

Despite these similarities, Ss and Ahr have very different roles in vivo. Ahr controls the physiological response to a variety of environmental toxins. Toxic aryl hydrocarbons such as dioxin bind to Ahr, and cause it to activate transcription of genes that encode the enzymes involved in toxin metabolism. Ahr knockout mutations in the mouse block this response to toxic compounds (Fernandez-Salguero et al., 1995; Schmidt et al., 1996). However, with the exception of defects in the liver and immune system (Fernandez-Salguero et al., 1995; Schmidt et al., 1996), these mutants do not show developmental abnormalities. In contrast, ss has been implicated only in developmental roles (Struhl, 1982; Burgess and Duncan, 1990; Duncan et al., 1998). Although it cannot be ruled out that ss plays some role in toxin metabolism, its restricted expression argues against any such role. Moreover, it seems unlikely that Ss binds dioxin, since key residues required for the binding of dioxin to Ahr are not conserved in Ss (Duncan et al., 1998). Consistent with this, dioxin binding activity has not been observed in arthropods or other invertebrates, although it is found in most vertebrates (Hahn et al., 1994). Also, direct tests indicate that the C. elegans ortholog of Ahr does not bind dioxin (Powell-Coffman et al., 1998).

Although it is unlikely that Ss binds dioxin, the extensive similarities between Ss and Ahr raise the question of whether Ss depends upon the binding of some other ligand for function. We have addressed this question by driving ectopic expression of $s s$ in the mesoderm (driven by $t w i$-GAL4) and in the ectoderm (driven by en-GAL4). In both locations, this causes coincident nuclear localization of Tgo. Thus, it would appear that the Ss-Tgo interaction does not require additional, tissue specific, signals. Although this observation argues against any requirement for a spatially restricted ligand, it leaves open the possibility that Ss interacts with some broadly distributed ligand. In addition, the inability to detect nuclear Tgo in the embryonic leg primordia that express ss could reflect differences in how Ss functions in different cell types.

A striking difference between Arnt and Tgo is that, in the absence of known bHLH-PAS partners, Arnt is generally found in the nucleus (Pollenz et al., 1994), whereas Tgo is localized to the cytoplasm (Ward et al., 1998). Consistent with this behavior, Arnt has a nuclear localization sequence near its $\mathrm{N}$ terminus, whereas Tgo does not (Eguchi et al., 1997). Indeed, all of the vertebrate Arnt-related proteins known have such a sequence, whereas neither the Drosophila nor C. elegans homologs do (B. Mitchell and S. T. Crews, unpublished data). This suggests that there may be a fundamental difference in how Arnt functions in vertebrates and invertebrates.

How did Ss and Ahr come to have such different functions in vertebrates and arthropods? One possibility is that Ahr functioned as some type of chemosensory protein in an ancestral organism. In vertebrates, this function became utilized by all cells to sense aryl hydrocarbon toxins, whereas in arthropods it became intimately associated with the specification of a major chemosensory organ, the antenna. It is hoped that studies of organisms from other lineages will shed light on how Ss and Ahr came to adopt such different roles.

The authors would like to thank Artyom Kopp and Mark Kankel for critically reviewing the manuscript, the UNC Department of Biology for use of their confocal microscope, Shin-Pei Shih for the Septin1 bait, Yue Ma for pAct5CSRS, and Ken Burtis for information regarding $\mathrm{pAct}$ 5CSRS. This work was supported by a Canadian MRC fellowship to M. S., an American Cancer Society fellowship to M. W., NICHD grant RD25251 and NSF grant IBN-9630381 to S. T. C., and NIH grant GM32318 to I. D.

\section{REFERENCES}

Brent, R. (1994). Interaction trap/two-hybrid system to identify interacting proteins. Curr. Prot. Mol. Biol. 27, 1-17.

Burgess, E. A. and Duncan, I. (1990). Direct control of antennal identity by the spineless-aristapedia gene of Drosophila. Mol. Gen. Genet. 221, 347352.

Burtis, K. C. and Baker, B. S. (1989). Drosophila doublesex gene controls somatic sexual differentiation by producing alternatively spliced mRNAs encoding related sex-specific polypeptides. Cell 56, 997-1010.

Carver, L. A. and Bradfield, C. A. (1997). Ligand-dependent interaction of the aryl hydrocarbon receptor with a novel immunophilin homolog in vivo. J. Biol. chem. 272, 11452-11456.

Coumailleau, P., Poellinger, L., Gustafson, J.-A. and Whitelaw, M. L. 1995. Definition of a minimal domain of the dioxin receptor that is associated with hsp90 and maintains wild-type ligand binding affinity and specificity. $J$. Biol. Chem. 270, 25291-25300.

Crews, S. T. (1998). Control of cell lineage-specific development and transcription by bHLH-PAS proteins. Genes Dev. 12, 607-620.

Denis, M., Cuthill, S., Wikstrom, A. C., Poellinger, L. and Gustafsson, J.A. (1988). Association of the dioxin receptor with the Mr 90,000 heat shock protein: a structural kinship with the glucocorticoid receptor. Biochem. Biophys. Res. Commun. 155, 801-807.

Denison, M. S., Fisher, J. M. and Whitlock, J. P. (1988). The DNA recognition site for the dioxin-Ah receptor complex. J. Biol. Chem. 263, 17221-17224.

Dolwick, K. M., Swanson, H. I., and Bradfield, C. A. (1993). In vitro analysis of $\mathrm{Ah}$ receptor domains involved in ligand-activated DNA recognition. Proc. Natl. Acad. Sci. USA 90, 8566-8570.

Duncan, I. M. (1982). Polycomblike: A gene that appears to be required for the normal expression of the bithorax and Antennapedia gene complexes of Drosophila melanogaster. Genetics 102, 49-70.

Duncan, D. M., Burgess, E. A. and Duncan, I. (1998). Control of distal antennal identity and tarsal development in Drosophila by spineless-aristapedia, a homolog of the mammalian dioxin receptor. Genes Dev. 12, 1290-1303.

Eguchi, H., Ikuta, T., Tachibana, T., Yoneda, Y. and Kawajiri, K. (1997) A nuclear localization signal of human aryl hydrocarbon receptor nuclear translocator/hypoxia-inducible factor $1 b$ is a novel bipartite type recognized by the two components of nuclear pore-targeting complex. J. Biol. Chem. 272, 17640-17647.

Fares, H., Peifer, M. and Pringle, J. R. (1995). Localization and possible functions of Drosophila septins. Mol. Biol. Cell 6, 1843-1859.

Fehon, R. G., Kooh, P. J., Rebay, I., Regan, C. L., Xu, T., Muskavitch, M. A. and Artavanis-Tsakonas, S. (1990). Molecular interactions between the protein products of the neurogenic loci Notch and Delta, two EGFhomologous genes in Drosophila. Cell. 61, 523-534. 
Fernandez-Salguero, P., Pineau, T., Hilbert, D. M., McPhail, T., Lee, S. S. T., Kimura, S., Nebert, D. W., Rudikoff, S., Ward, J. M. and Gonzalez, F. J. (1995). Immune system impairment and hepatic fibrosis in mice lacking the dioxin-binding Ah receptor. Science 268, 722-726.

Fields, S. and Song, O. (1989). A novel genetic system to detect proteinprotein interactions. Nature 340, 245-246.

Gekakis, N., Saez, L., Delahaye-Brown, A. M., Myers, M., Sehgal, A., Young, M. W., and Weitz, C. J. (1995). Isolation of timeless by PER protein interaction: Defective interaction between timeless protein and longperiod mutant $\mathrm{PER}^{\mathrm{L}}$. Science 270, 811-815.

Hahn, M. E., Poland, A., Glover, E. and Stegement, J. J. (1994) Photoaffinity labeling of the Ah receptor: phylogenetic survey of diverse vertebrate and invertebrate species. Arch. Biochem. Biophys. 310, 218-228.

Han, K., Levine, M. S. and Manley, J. L (1989). Synergistic activation and repression of transcription by Drosophila homeobox proteins. Cell 56, 573583.

Hankinson, O. (1995). The Aryl hydrocarbon receptor complex. Ann. Rev. Pharmacol. Toxicol. 35, 307-40.

Huang, Z. J., Edery, I., and Rosbash, M. (1993). PAS is a dimerization domain common to Drosophila period and several transcription factors. Nature 364, 259-262.

Isaac, D. D. and Andrew, D. J. (1996). Tubulogenesis in Drosophila: a requirement for the trachealess gene product. Genes Dev. 10, 103-117.

Lindebro, M. C., Poellinger, L. and Whitelaw, M. L. (1995). Protein-protein interaction via PAS domains: role of the PAS domain in positive and negative regulation of the bHLH/PAS dioxin receptor-Arnt transcription factor complex. EMBO J. 101-112.

Lindsley, D. L. and Zimm, G. G. (1992). The Genome of Drosophila melanogaster. Academic Press, San Diego, CA.

Ma, Q. and Whitlock, J. J. P. (1997). A novel cytoplasmic protein that interacts with the ah receptor, contains tetratricopeptide repeat motifs, and augments the transcriptional response to 2, 3, 7, 8-tetrachlorodibenzo-pdioxin. J. Biol. Chem. 272, 8878-8884

Michaud, J. and Fan, C. M. (1997). Single-minded - two genes, three chromosomes. Genome Res. 7, 569-71.

Michaud, J. L., Rosenquist, T., May, N. R. and Fan, C. M. (1998). Development of neuroendocrine lineages requires the bHLH-PAS transcription factor SIM1. Genes Dev. 12, 3264-3275.

Nambu, J. R., Chen, W., Hu, S. and Crews, S. T. (1996). The Drosophila melanogaster similar bHLH-PAS gene encodes a protein related to human Hypoxia-inducible factor $1 a$ and Drosophila Single-minded. Gene 172, 249254

Nambu, J. R., Franks, R. G., Hu, S. and Crews, S. T. (1990). The singleminded gene of Drosophila is required for the expression of genes important for the development of CNS midline cells. Cell 63, 63-75.

Nambu, J. R., Lewis, J. L., Wharton, K. A. and Crews, S. T. (1991). The Drosophila single-minded gene encodes a helix-loop-helix protein which acts as a master regulator of CNS midline development. Cell 67, 11571167.

Ohshiro, T. and Saigo, K. (1997). Transcriptional regulation of breathless FGF receptor gene by binding of TRACHEALESS/dARNT heterodimers to three central midline elements in Drosophila developing trachea. Development 124, 3975-3986.

Patel, N. H., Snow, P. M. and Goodman, C. S. (1987). Characterization and cloning of fasciclin III: a glycoprotein expressed on a subset of neurons and axon pathways in Drosophila. Cell 48, 975-988.
Pollenz, R. S., Sattler, C. A. and Poland, A. (1994). The aryl hydrocarbon receptor and aryl hydrocarbon receptor nuclear translocator protein show distinct subcellular localizations in Hepa $1 \mathrm{c} 1 \mathrm{c} 7$ cells by immunofluorescence microscopy. Mol. Pharmacol. 45, 428-438.

Pongratz, I., Antonsson, C., Whitelaw, M. L. and Poellinger, L. (1998) Role of the PAS domain in regulation of dimerization and DNA binding specificity of the dioxin receptor. Mol. Cell. Biol. 18, 4079-4088.

Pongratz, I., Strömstedt, P. E., Mason, G. G. and Poellinger, L. (1991). Inhibition of the specific DNA binding activity of the dioxin receptor by phosphatase treatment. J. Biol. Chem. 266, 16813-16817.

Powell-Coffman, J. A., Bradfield, C. A. and Wood, W. B. (1998). Caenorhabditis elegans orthologs of the aryl hydrocarbon receptor and its heterodimerization partner the aryl hydrocarbon receptor nuclear translocator. Proc. Natl. Acad. Sci. USA 95, 2844-2849.

Reisz-Porszasz, S., Probst, M. R., Fukunaga, B. N. and Hankinson, O. (1994). Identification of functional domains of the aryl hydrocarbon receptor nuclear translocator protein (ARNT). Mol. Cell. Biol. 14, 6075-6086.

Rowlands, J. C. and Gustafsson, J.-A. (1997). Aryl hydrocarbon receptormediated signal transduction. Crit. Rev. Toxicol. 27, 109-134.

Schmidt, J. V., Su, H-T., Reddy, J. K., Simon, M. C. and Bradfield, C. A. (1996). Characterization of a murine $A h r$ null allele: involvement of the Ah receptor in hepatic growth and development. Proc. Natl. Acad. Sci. USA 93, 6731-6736.

Schmidt, J. V. and C. A. Bradfield. (1996). Ah receptor signaling pathways. Ann. Rev. Cell Dev. Biol. 12, 55-89.

Semenza, G. L. (1998). Hypoxia-inducible factor 1: master regulator of O2 homeostasis. Curr. Opin. Genet. Dev. 8, 588-594.

Sonnenfeld, M., Ward, M., Nystrom, G., Mosher, J., Stahl, S. and Crews, S. (1997). The Drosophila tango gene encodes a bHLH-PAS protein that is orthologous to mammalian Arnt and controls CNS midline and tracheal development. Development 124, 4583-4594.

Struhl, G. (1982). spineless-aristapedia: A homeotic gene that does not control the development of specific compartments in Drosophila. Genetics 102, 737-749.

Tautz, D. and Pfeiffle, C. (1989). A nonradioactive in situ hybridization method for the localization of specific RNAs in Drosophila embryos reveals translational control of the segmentation gene hunchback. Chromosoma $\mathbf{9 8}$ 81-85.

Thomas, J. B., Crews, S. T. and Goodman, C. S. (1988). Molecular genetics of the single-minded locus: a gene involved in the development of the Drosophila nervous system. Cell 52, 133-141.

Ward, M. P., Mosher, J. T. and Crews, S. T. (1998). Regulation of Drosophila bHLH-PAS protein cellular localization during embryogenesis. Development 125, 1599-1608.

Wharton, J., K. A., Franks, R. G., Kasai, Y. and Crews, S. T. (1994) Control of CNS midline transcription by asymmetric E-box elements: similarity to xenobiotic responsive regulation. Development 120, 3563 3569.

Wilk, R., Weizman, I., Glazer, L. and Shilo, B.-Z. (1996). trachealess encodes a bHLH-PAS protein and is a master regulator gene in the Drosophila tracheal system. Genes Dev. 10, 93-102.

Xu, T. and Rubin, G. M. (1993). Analysis of genetic mosaics in developing and adult Drosophila tissues. Development 117, 1223-1237.

Zelzer, E., Wappner, P. and Shilo, B.-Z. (1997). The PAS domain confers target gene specificity of Drosophila bHLH/PAS proteins. Genes Dev. 11, 2079-2089. 